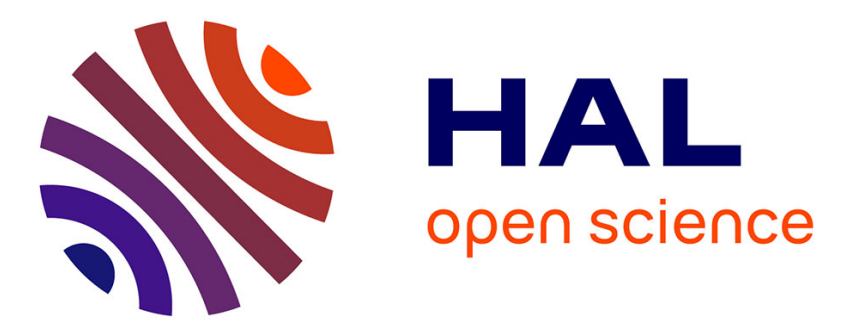

\title{
Twisted pseudo-tetragonal orthorhombic lamellar crystal in cellulose/ionic liquid spherulite
}

Guangjie Song, Jun Zhang, Yoshiharu Nishiyama

\section{To cite this version:}

Guangjie Song, Jun Zhang, Yoshiharu Nishiyama. Twisted pseudo-tetragonal orthorhombic lamellar crystal in cellulose/ionic liquid spherulite. Cellulose, 2020, 27 (10), pp.5449-5455. 10.1007/s10570020-03214-6 . hal-02951280

\author{
HAL Id: hal-02951280 \\ https://hal.science/hal-02951280
}

Submitted on 12 Oct 2021

HAL is a multi-disciplinary open access archive for the deposit and dissemination of scientific research documents, whether they are published or not. The documents may come from teaching and research institutions in France or abroad, or from public or private research centers.
L'archive ouverte pluridisciplinaire HAL, est destinée au dépôt et à la diffusion de documents scientifiques de niveau recherche, publiés ou non, émanant des établissements d'enseignement et de recherche français ou étrangers, des laboratoires publics ou privés. 


\title{
1 Twisted pseudo-tetragonal orthorhombic \\ 2 lamellar crystal in cellulose/ionic liquid \\ 3 spherulite
}

\author{
4 Guangjie Song ${ }^{\mathrm{a}}$, Jun Zhang ${ }^{\mathrm{a} *}$, Yoshiharu Nishiyama ${ }^{\mathrm{b}}$ * \\ 5 a Beijing National Laboratory for Molecular Sciences, CAS Key Laboratory of \\ 6 Engineering Plastics, Institute of Chem-istry, Chinese Academy of Sciences, \\ 7 Beijing 100190, China \\ 8 b Univ. Grenoble Alpes, CNRS, CERMAV, 38000 Grenoble \\ 9 \\ 10 Corresponding Author \\ $11 *$ Email: jzhang@iccas.ac.cn \\ 12 *Email: yoshiharu.nishiyama@cermav.cnrs.fr \\ 13 ORCID \\ 14 Guangjie Song: 0000-0002-3329-4629 \\ 15 Jun Zhang: 0000-0003-4824-092X \\ 16 Nishiyama Yoshiharu: 0000-0003-4069-2307

18 ABSTRACT: Cellulose and Ionic liquids can form new types of complex crystals growing in form

19 of spherulites. Microfocus X-ray diffraction on selected location of a spherulite, formed from

20 cellulose solution in 1-allyl-3-methylimidazolium chloride $(\mathrm{AmimCl})$ by water vapor diffusion,

21 gave diffraction patterns of fiber-type texture that could be used to index the diffraction spots. The

22 unit cell dimension was tetragonal with $a=b=12.92 \AA, c=10.4 \AA$, at room temperature and $a=$

$23 b=12.8 \AA$ and $c=10.45 \AA$ at $100 \mathrm{~K}$ with $\alpha=\beta=\gamma=90^{\circ}$. However, the symmetry of the molecule

24 and stoichiometry suggests a space group $P 2{ }_{1} 2_{1} 2_{1}$, compatible with chiral chain molecule with the

25 systematic absence of odd-order h $00,0 \mathrm{k} 0$, and 001 reflections. The spherulite consists of

26 lamellar crystallites rotating along the $\mathrm{b}$ axis, the latter lying in the radial direction. The half pitch

27 of the order of $12 \mu \mathrm{m}$ according to polarized microscopy, was smaller than the beam size leading

28 to a fiber-like pattern. The crystallite sizes estimated from the diffraction broadening were at least

29 of the order of $150 \mathrm{~nm}$ along a and b direction, limited by the instrument resolution, and about

$3015 \sim 30 \mathrm{~nm}$ along c direction. The $10.4 \AA$ of $c$-axis is the typical length of two-fold helix of

31 cellulose suggesting that cellulose chain is parallel to the c-axis. The unit cell would contain 4

32 glucose residues, 4 ionic liquid molecules and $4-8$ water molecules.

33 Keywords: Cellulose, Ionic liquid, Spherulite, Complex crystal, X-ray diffraction 


\section{Introduction}

2 Understanding the interaction of solvent and polymer is essential in optimized

3 polymer processing and design of solvents (Guenet 2008). When crystallosolvate

4 is available, crystal structure analysis allows direct inspection of spatial

5 arrangement of polymer and small molecules leading to better understanding the

6 interaction (Noishiki et al. 2003; Sawada et al. 2013a; Sawada et al. 2012; Sawada

7 et al. 2013b; Wada et al. 2008; Wada et al. 2006). For example, cellulose or its

8 analogues can form complex crystal with different types of amine without losing

9 its crystallinity, and in some particular case with increased crystallinity. The

10 general tendency of amine to coordinate to the primary alcohol group, with

11 hydroxyl groups donating to amine, has been established, or sometimes water

12 molecule disrupting intermolecular hydrogen bond in chitin (Sawada et al. 2014;

13 Sawada et al. 2012; Sawada et al. 2016).

14 Room-temperature ionic liquids (ILs) such as 1-allyl-3-methylimidazolium

15 chloride (AmimCl) and 1-butyl-3-methylimidazolium chloride (BmimCl) hold

16 great potential as green solvents for cellulose dissolution because of their excel-

17 lent dissolving capability, negligible vapor pressure, high thermal stability, and

18 ease of recycling (Rigual et al. 2017; Zhang et al. 2005). We investigated the

19 crystallization behavior of cellulose in ionic liquid solution through water-vapor-

20 diffusion precipitation, and found that the crystalline part in the spherulite is a

21 cellulose/ionic liquid complex crystal giving very sharp powder diffraction

22 patterns unusual for cellulose (Song et al. 2018). However, the unit cell could not

23 be determined in the absence of indexation of diffraction peaks that are shrunk

24 into $1 \mathrm{D}$ data in a powder pattern.

25 Here we further performed X-ray diffraction on a single spherulite to obtain

26 textured pattern of 2D nature to help the assignment of the peaks and get

27 structural insights on the spherulite.

29 Experimental

30 Cellulose spherulites were prepared from cellulose/ionic liquid solution

31 according to the method previously reported (Song et al. 2018). In short,

32 microcrystalline cellulose (degree of polymerization $~ 220$ ) was dissolved in 
1 AmimCl to make $19 \%$ solution. The solution was cast on a glass slide, preheated

2 to $100^{\circ} \mathrm{C}$ to form a thin layer and covered with a cover glass and held at $100{ }^{\circ} \mathrm{C}$

3 for $10 \mathrm{~min}$ to eliminate possible effects of thermal history. Then the temperature

4 was reduced to $60{ }^{\circ} \mathrm{C}$. The whole setup was encased in a purged dry chamber to

5 avoid contact with humidity during this process. Then, water vapor was diffused

6 into the chamber and maintained at $10 \%$ relative humidity leading to the

7 formation of spherulites. Individual spherulites embedded in the films were

8 collected with tweezers.

9 X-ray diffraction measurement on the spherulite was performed at beamline

10 17B at Shanghai Synchrotron Radiation Facility. The sample was mounted on a

11 loop fixed on a goniometer head that was translated in horizontal and vertical

12 direction perpendicular to the beam using a motorized stage. An optical

13 microscope CCD camera, calibrated for the beam position, was used to precisely

14 locate the spherulite with respect to the incident beam. A $50 \mu \mathrm{m}$ x $50 \mu \mathrm{m}$ beam

15 and a wavelength of $0.98 \AA$ was used with an exposure time of 100 seconds. The

16 scattering was recorded using a MX300 detector (pixel size $=146 \mu \mathrm{m}$ ) at a

17 specimen-to-detector distance of $250 \mathrm{~mm}$.

18 AmimCl was crystallized by overcooling and a single crystal was cut off under

19 a microscopy. Single-crystal X-ray analysis of the AmimCl single crystal was

20 performed on the Rigaku MM007 HF diffractometer on a rotating anode (Mo-K

21 radiation, $0.71073 \AA$ ) at $100 \mathrm{~K}$. The structure was solved by the direct method

22 using SHELXT and refined with SHELXL. The crystallographic data is reported

23 in supplementary information. Besides, this crystal structure data has been

24 deposited in the Cambridge Crystallographic Data Base as CCDC-1981478.

\section{Results and discussion}

27 A room temperature X-ray diffraction pattern, measured from region close to

28 the border of a large spherulite, is shown in Fig. 1. The digital image of the

29 spherulite and the beam position are shown in the inset. The range of size of the

30 observed and analyzed spherulites is about $0.5 \sim 1 \mathrm{~mm}$. The diffraction pattern

31 shows clearly separated arcs with a symmetry with respect to an axis radial to the

32 spherulite (blue line in Fig. 1). The diffraction closest to the center are four arcs

33 (red circles) centered at about $+/-45^{\circ}$ with respect to the blue line axis, followed 
1 by another four arcs (red arrows) that are parallel and perpendicular to the blue

2 line axis. The d-spacings of the first 4 diffraction spots were $9.14 \AA$ while that of

3 the next 4 spots were $6.46 \AA$ corresponding to a d-spacing ratio of $\sqrt{ } 2: 1$. This

4 implies already a square lattice $a=b=12.92 \AA$ and $\gamma=90^{\circ}$. However, the

5 assignment of many other spots is less straightforward. Further inspection of the

6 image gives an impression of small deviation from the symmetry around an axis

7 perpendicular to the blue line. For example, a peak is split along the azimuthal

8 line, merging on the opposite side, and the intensities along the line are not

9 symmetric (cf. Blue ellipse in Fig. 1). This kind of feature is typical of tilted fiber

10 diffraction where the fiber axis is along blue line.

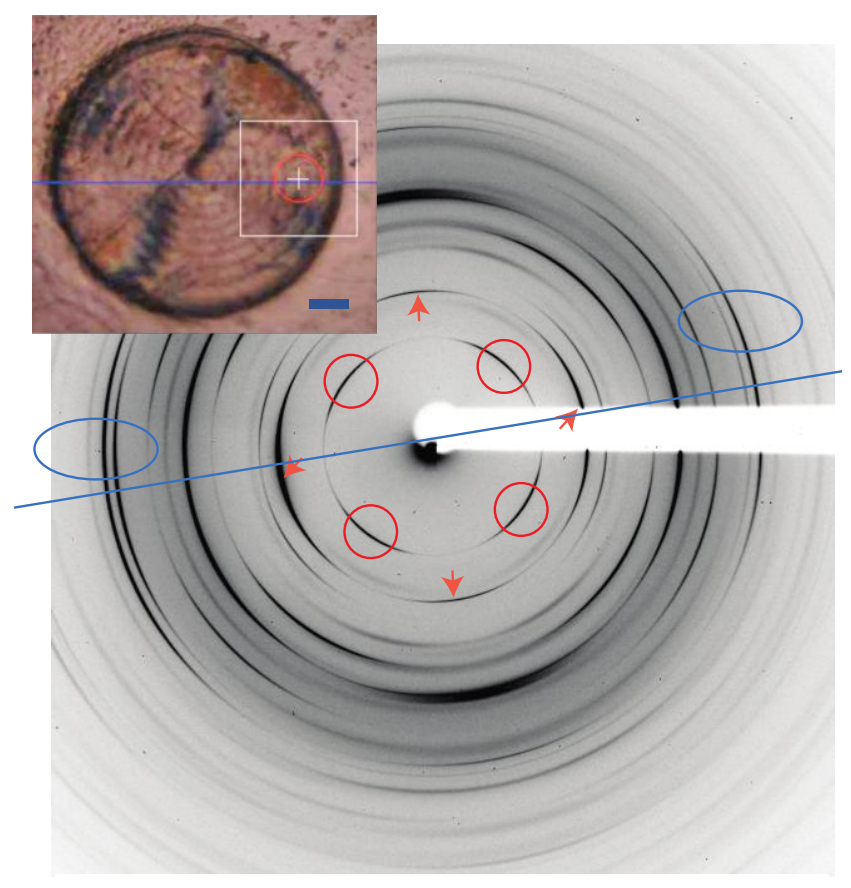

Fig. 1 2D X-ray diffraction image taken close to the border of a cellulose-ionic liquid spherulite. Inset: Digital image of the spherulite taken with an optical microscope at the beam position $($ scale bar $=100 \mu \mathrm{m})$

11 We thus remapped the intensity into a projection of cylindrical reciprocal

12 coordinate system adjusting the assumption of fiber tilt to obtain more symmetric

13 pattern, using an in-house software (Fig. S1, Supplementary Information). Then

14 we varied systematically the unit cell parameter c assuming $\alpha=\beta=90^{\circ}$, to index

15 all spots on the binned pattern, and obtained $c=10.4 \AA$ as smallest parameter that

16 can explain all diffraction spots as shown in Fig. 2. This length is roughly the 
1 same as fiber repeat of all cellulose allomorphs with two-fold helical

2 conformation.

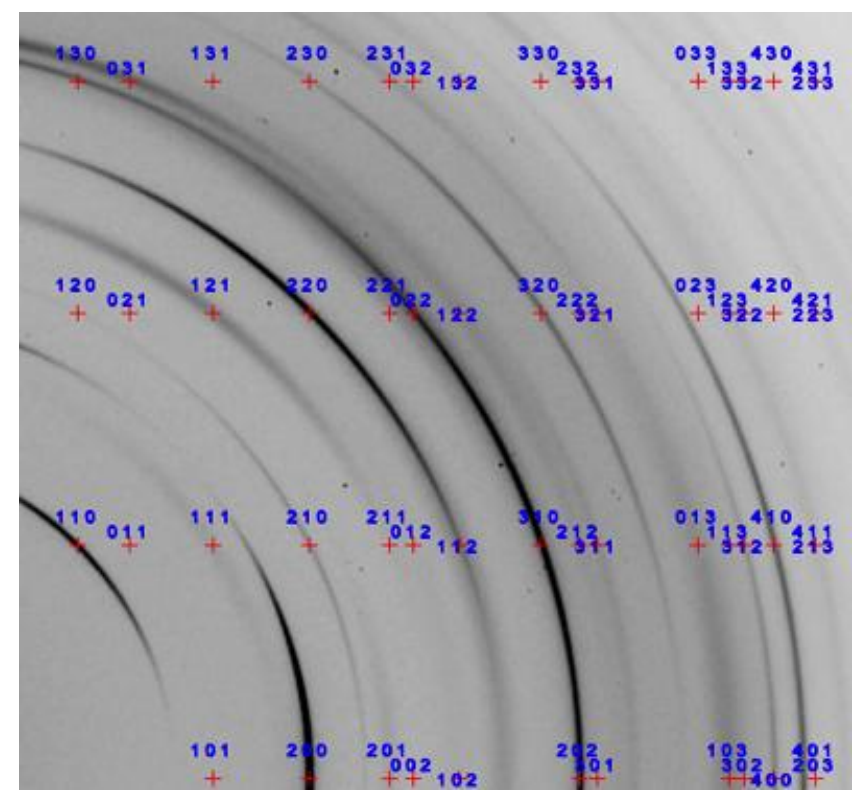

Fig. 2 2D X-ray diffraction image of Fig. 1 remapped into cylindrical reciprocal space coordinate, where the fiber axis (center of symmetry) is vertical with lattice points in red corresponding to the Miller indices in blue

3 The lattice parameters obtained here would imply a tetragonal unit cell. The 4 unit cell volume of $1730 \AA^{3}$ can accommodate 8 glucose residues, but this would

5 leave no space for ionic liquid molecules with symmetry operation. Thus, a

6 reasonable number of glucose residues in the unit cell would be 4 , limiting the

7 number of asymmetric glucose unit to one, and symmetry operation to 4 . There

8 are 6 chiral space groups for tetragonal unit cell: $P 4, P 4_{1}, P 4_{2}, P 4_{3}, I 4$ and $I 4_{1} . I 4$

9 and $I 4_{1}$ are discarded because they have 8 symmetry operations. $\mathrm{P} 4$ and $\mathrm{P} 4_{2}$

10 cannot make a polymer because the symmetry $x, y, z$ and $-x,-y, z$ collide when

11 placed on the symmetry axis. $P 4_{1}$ and $P 4_{3}$ are possible candidates for

12 polysaccharides in general, but the rigid glucose residue and the conformational

13 space allowed for the glycosidic torsion angles does not allow a 4-fold helix with

14 a $10 \AA$ period (French 1985; French 2012). Thus, the tetragonal space groups were 15 discarded.

16 An alternative is an orthorhombic unit cell which is very close to tetragonal,

17 and in this case only $P 2{ }_{1} 2_{1} 2_{1}$ symmetry allows the construction of chain with two

18 fold helix without collision. The odd-order reflections h $00,0 \mathrm{k} 0$ or 001

19 prohibited by the symmetry are indeed absent. This unit cell and space group

20 combination is also in agreement with the solid-state NMR data that gives only 
1 one resonance peak for each carbon of glucose. The volume of glucose residue

2 can be estimated from the unit cell volumes of pure cellulose allomorphs, as

3 between $164.5 \AA^{3}$ (cellulose I) to $173.8 \AA^{3}$ (cellulose $\mathrm{III}_{\mathrm{I}}$ ). To estimate the

4 molecular volume of $\mathrm{AmimCl}$, we measured the density of liquid phase by using a

5 Kratky type digital density meter (DM45 DeltaRange Mettler Toledo) and

6 obtained of $1.1491 \mathrm{~g} / \mathrm{cm}^{3}$ at $20{ }^{\circ} \mathrm{C}$. Using the formula weight of 158.6 the volume

7 of AmimCl can be calculated as $229 \AA^{3}$. Alternatively, single crystals of neat

$8 \mathrm{AmimCl}$ had a unit cell volume of $1641 \AA^{3}$ at $100 \mathrm{~K}$ containing 8 molecules,

9 giving a small limit of $205 \AA^{3}$.

10 If all molecules occupy crystallographic positions with an occupancy of 1 in

11 the space group $P 22_{1} 2_{1} 2_{1}$, the number of molecules would be multiple of 4 . If we

12 take the smallest estimations, the volume occupied by 4 glucose residues and 4

$13 \mathrm{AmimCl}$ is $1478 \AA^{3}$ leaving space for eight water molecules occupying $30 \AA^{3}$

14 each. On the other end, using the higher end, 4 glucose residues and $4 \mathrm{AmimCl}$

15 would occupy $1611 \AA^{3}$ leaving space for 4 water molecules. Both cases fit almost

16 exactly to the measured unit cell volume. The stoichiometry of glucose to

$17 \mathrm{AmimCl}$ of $1: 1$ is in good agreement with resonance intensity ratio in solid state

18 NMR spectra reported before.

19 Based on the above assumption of unit cell composition, the density of the

20 cellulose - AmimCl complex crystal would lie between $1.35 \mathrm{~g} / \mathrm{cm}^{3}$ (4 water

21 molecules) and $1.46 \mathrm{~g} / \mathrm{cm}^{3}$ (8 water molecules). We evaluated the density of the

22 spherulite, by floating method using a series of mixture of CCl4/Acetone, to be

23 between 1.246 and $1.270 \mathrm{~g} / \mathrm{cm}^{3}$. The difference between the crystal density and

24 the spherulite can be attributed to the presence of cellulose depleted amorphous

25 component in the spherulite (Song et al. 2018), since the cellulose concentration

26 in the crystal is $43-46 \%$, much higher than the solution concentration of $19 \%$. So

27 the spherulite indeed should contain significant amount of amorphous.

28 In Fig. 2 and Fig. S1, there are peaks with different broadening in the radial

29 direction. In the meridional direction, most diffractions are sharp, but the

30 diffraction close to the equator has various broadening. To estimate the crystallite

31 size from the line broadening, diffraction data measured at $300 \mathrm{~mm}$ detector

32 distance and an X-ray wavelength of $1.2 \AA$ was used to minimize the instrument

33 broadening. Fig. 3 shows some of the line profiles obtained from the data

34 remapped into polar coordinate (Fig. S2, Supplementary Information). The data 


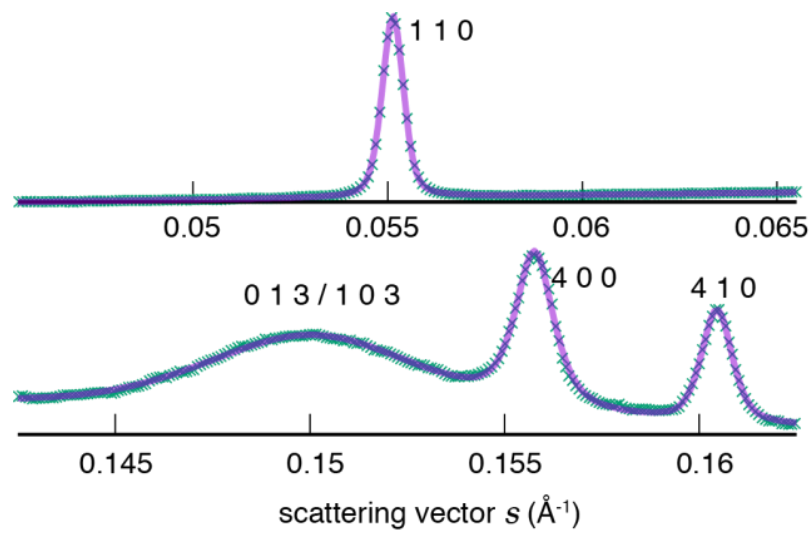

Fig. 3 Radial line profiles of selected diffraction spots as a function of scattering vector $\mathrm{s}=2 \sin \theta / \lambda$

1 was over-sampled along radial direction by a factor of two to keep the resolution.

2 Each point corresponds to a half-pixel, so the peak width of 110 on the detector

3 corresponds to only a few pixels. Still, the line broadening would give the smaller

4 bound of the crystallite size. The diffraction peaks were fitted using pseudo-Voigt

5 functions (Wertheim et al. 1974) on a linear baseline and the resulting parameters

6 and corresponding apparent crystallite sizes are reported in table 1. All diffraction

7 spots in the direction close to the $c^{*}$ axis are relatively broad, meaning that the

8 crystals are flat and expanding in the a-b plane. Also it can be seen that the

9 apparent crystallite size are smaller for higher order diffraction (higher angles)

10 though the contribution of instrument broadening is smaller. This indicates the

11 presence of disorder of second kind, i.e. paracrystals (Lindenmeyer and

12 Hosemann 1963).

Table 1 apparent crystallite sizes from the line

\begin{tabular}{|c|c|c|c|c|}
\hline $\begin{array}{l}\text { miller } \\
\text { indices }\end{array}$ & $\begin{array}{l}\text { scattering } \\
\text { vector }\left(\mathrm{nm}^{-1}\right)\end{array}$ & $\begin{array}{l}\text { peak width } \\
\left(\mathrm{nm}^{-1}\right)^{\mathrm{a}}\end{array}$ & $\begin{array}{l}\text { shape } \\
\text { parameter }^{b}\end{array}$ & $\begin{array}{l}\text { crystallite } \\
\text { size }(\mathrm{nm})\end{array}$ \\
\hline 110 & 0.55 & 0.0065 & 0.64 & 155 \\
\hline 200 & 0.78 & 0.0068 & 0.49 & 146 \\
\hline 210 & 0.87 & 0.0070 & 0.66 & 142 \\
\hline 220 & 1.10 & 0.0090 & 0.27 & 111 \\
\hline 400 & 1.56 & 0.0117 & 0.30 & 85 \\
\hline 410 & 1.60 & 0.0093 & 0.53 & 108 \\
\hline 111 & 0.73 & 0.0333 & 0.89 & 30 \\
\hline 121 & 0.995 & 0.0256 & 0.95 & 39 \\
\hline 013 & 1.5 & 0.0654 & 0.7 & 15 \\
\hline 103 & & & & \\
\hline
\end{tabular}


1 The crystallites sizes are shown in as vector length in Fig. 4. The crystallites are

2 at least $150 \mathrm{~nm}$ wide and $15-30 \mathrm{~nm}$ thick probably forming twisted lamellae

3 along the radial direction with a pitch smaller than the X-ray beam size (50 $\mu \mathrm{m})$

4 so that a fiber pattern can be obtained. The optical micrograph in Fig. 1 is indeed

5 showing a stripe pattern typical of such twisted structure.

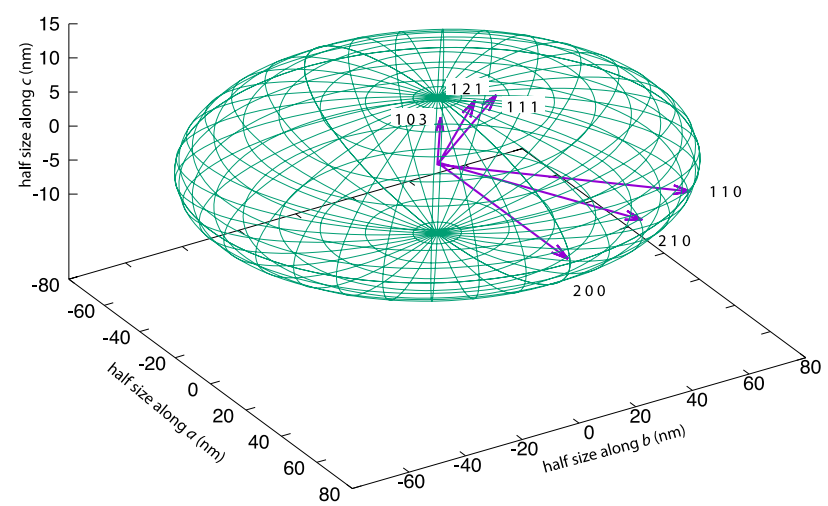

Fig. 4 Crystallite size along different direction. The half-size is indicated as vector length from the origin in the direction in the reciprocal space. The ellipsoid approximates the crystallite shape

6 The chain length of cellulose used for crystallization is much longer than the

7 thickness along c-axis direction (15 30 nm) might imply chain folding in the

8 complex crystal (Yamane et al. 2013). However, the amount of cellulose in the

9 amorphous state is not clear. If the amorphous fraction is high, the chains can re-

10 enter the crystal with long loop in amorphous region or form fringed micelle. The

11 fact that the chains in the crystal are laterally separated by ionic liquid would also

12 facilitate the chain connection through amorphous part avoiding a steep folding. If

13 the major part of cellulose chains is in crystalline state, the chain has to fold

14 relatively tightly. The CP-MAS NMR in our previous report might have not

15 captured whole amorphous fraction if they are highly mobile. A further

16 quantitative NMR experiment would clarify this point in the future.

17 We further investigated the spherulite under crossed polarizers without and

18 with a full-wave retardation plate $(530 \mathrm{~nm})$. The spherulites grown from thick

19 films showed Maltese cross with band structures with different colors. Fig. 5a

20 shows a typical example in this case with alternating yellowish and gray bands for

21 a thickness of $43 \mu \mathrm{m}$. The same bands, after insertion of the retardation plate,

22 changed to yellow and light blue in the north-west and south-east quadrants, and 
1 orange and yellowish in the north-east and south-west quadrants. This can be

2 explained by the retardation of about 300 and $100 \mathrm{~nm}$ that adds to the $530 \mathrm{~nm}$ in

3 the NW, SE quadrants and subtracted in the NE, SW quadrants, indicating that the

4 major axis of birefringence is in tangential direction (negative spherulite).

(A)

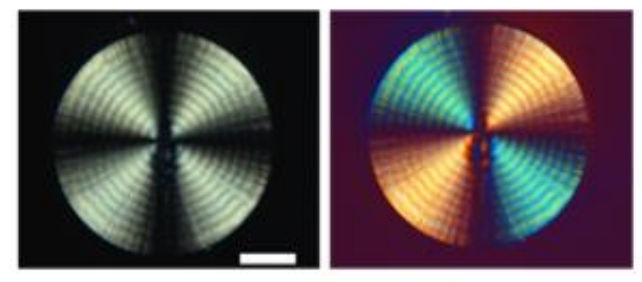

(B)
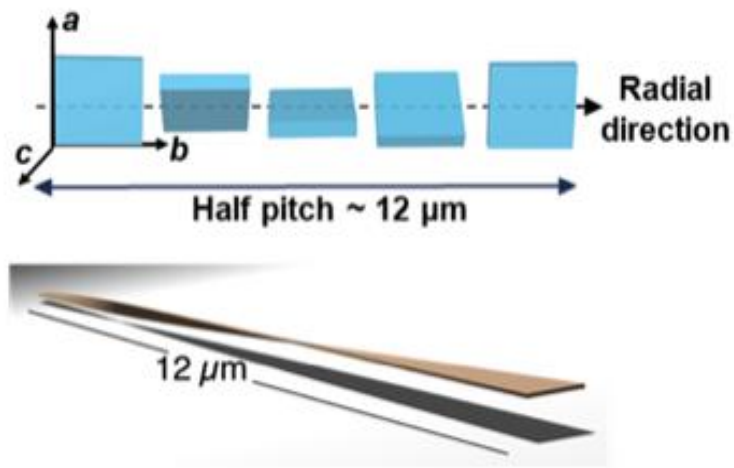

Fig. 5 (A) A POM image without and with a full-wave retardation plate $(530 \mathrm{~nm})$ (scale bar $=100 \mu \mathrm{m})$; (B) schematic illustration of lamellar crystal in spherulite with twists

$5 \quad$ The strong birefringence in the $c b$ plane can be due to the intrinsic

6 birefringence of cellulose chain. If the banded feature is due to twisting (Fig. 5b),

7 the fact that in both bands of strong and weak birefringence show negative

8 birefringence means that, the crystals probably show birefringence in ab plane too

9 with a-axis being the major axis. The intrinsic birefringence of the domains

10 judging from the Newton colors and thicknesses are of the order of 0.003 and 0.01

11 for the weak and strong birefringent regions respectively. The birefringence of

12 neat cellulose is about 0.07 , and thus the value of 0.01 is in reasonable range

13 taking into account the limited volume fraction of cellulose in the crystal (38\%),

14 limited crystallinity as a whole and the fact that the form birefringence would

15 negatively contribute (Iyer et al. 1968; Lasseuguette et al. 2008). As the

16 birefringence seems to be constant along the radius, the density of crystal would

17 be constant from the center to the periphery, and since the diffraction peaks had 
1 similar sharpness in the center (Song et al. 2018), the number of such plate-like

2 crystals are increasing towards the periphery.

\section{Conclusions}

5 The twisted pseudo-tetragonal orthorhombic lamellar crystal in cellulose/ionic

6 liquid spherulite was revealed in this study. The unit cell of the complex crystal

7 was tetragonal with $a=b=12.92 \AA, \mathrm{c}=10.4 \AA$, at room temperature and $a=b=$

$8 \quad 12.8 \AA$ and $c=10.45 \AA$ at $100 \mathrm{~K}$ with $\alpha=\beta=\gamma=90^{\circ}$ by microfocus X-ray

9 diffraction on selected location of an individual spherulite. The space group was

10 reasonably assigned as $P 2_{1} 2_{1} 2_{1}$ because of constraint of molecular symmetry and

11 stoichiometry. The crystallite sizes estimated from the diffraction broadening

12 were at least of the order of $150 \mathrm{~nm}$ along a and $\mathrm{b}$ direction, limited by the

13 instrument resolution, and about 15 30 $\mathrm{nm}$ along $\mathrm{c}$ direction. The spherulite

14 showed the major optic axis in tangential direction, giving rise to the intrinsic

15 birefringence of the order of 0.003 and 0.01 for the weak and strong birefringent

16 regions. The strong birefringence in the $c b$ plane can be due to the intrinsic

17 birefringence of cellulose chain.

\section{Acknowledgments}

20 This work was supported by the National Key Research and Development Program of China

21 (2017YFA0403103) and the National Natural Science Foundation of China (Nos. 21704107 and

22 51425307). We thank the staffs from BL17B beamline of National Facility for Protein Science

23 Shanghai (NFPS) at Shanghai Synchrotron Radiation Facility, for assistance during data

24 collection. The measurement of single-crystal X-ray diffraction was performed at the Center for

25 Physicochemical Analysis and Measurements in ICCAS. The help from Ms. Tongling Liang was

26 acknowledged.

\section{References}

29 French AD (1985) Physical and theoretical methods for determining the supramolecular structure

30 of cellulose. In: Nevell TP, Zeronian SH (eds) Cellulose Chemistry and its Applications. Ellis

31 Horwood, Chichester, pp 84-111 
1 French AD (2012) Chapter 2 - Combining Computational Chemistry and Crystallography for a

2 Better Understanding of the Structure of Cellulose. In: Horton D (ed) Advances in Carbohydrate

3 Chemistry and Biochemistry, vol 67. Academic Press, pp 19-93. doi:10.1016/B978-0-12-396527-

4 1.00002-4

5 Guenet J-M (2008) Polymer-Solvent Molecular Compounds. Elsevier, Amsterdam

6 Iyer KRK, Neelakantan P, Radhakrishnan T (1968) Birefringence of native cellulosic fibers. I.

7 Untreated cotton and ramie. J Polym Sci, Part A-2: Polym Phys 6:1747-1758

8 doi:10.1002/pol.1968.160061005

9 Lasseuguette E, Roux D, Nishiyama Y (2008) Rheological properties of microfibrillar suspension

10 of TEMPO-oxidized pulp. Cellulose 15:425-433 doi:10.1007/s10570-007-9184-2

11 Lindenmeyer PH, Hosemann R (1963) Application of the Theory of Paracrystals to the Crystal

12 Structure Analysis of Polyacrylonitrile. J Appl Phys 34:42-45 doi:10.1063/1.1729086

13 Noishiki Y, Nishiyama Y, Wada M, Okada S, Kuga S (2003) Inclusion Complex of $\beta$-Chitin and

14 Aliphatic Amines. Biomacromolecules 4:944-949 doi:10.1021/bm034024k

15 Rigual V, Santos TM, Domínguez JC, Alonso MV, Oliet M, Rodriguez F (2017) Recovery and

16 Reuse of 1-Allyl-3-methylimidazolium Chloride in the Fractionation of Pinus radiata Wood. ACS

17 Sustainable Chem Eng 5:2384-2392 doi:10.1021/acssuschemeng.6b02723

18 Sawada D, Hanson L, Wada M, Nishiyama Y, Langan P (2014) The initial structure of cellulose

19 during ammonia pretreatment. Cellulose 21:1117-1126 doi:10.1007/s10570-014-0218-2

20 Sawada D, Kimura S, Nishiyama Y, Langan P, Wada M (2013a) The crystal structure of mono-

21 ethylenediamine $\beta$-chitin from synchrotron X-ray fiber diffraction. Carbohydr Polym 92:1737-

221742 doi:10.1016/j.carbpol.2012.11.025

23 Sawada D, Nishiyama Y, Langan P, Forsyth VT, Kimura S, Wada M (2012) Water in Crystalline

24 Fibers of Dihydrate $\beta$-Chitin Results in Unexpected Absence of Intramolecular Hydrogen

25 Bonding. PLOS ONE 7:e39376 doi:10.1371/journal.pone.0039376

26 Sawada D et al. (2013b) Structure and dynamics of a complex of cellulose with EDA: insights into

27 the action of amines on cellulose. Cellulose 20:1563-1571 doi:10.1007/s10570-013-9974-7

28 Sawada D, Ogawa Y, Nishiyama Y, Togawa E, Kimura S, Langan P (2016) Molecular Interactions

29 in an $\alpha$-Chitin/Hydrazine Complex: Dynamic Hydrogen Bonds and Improvement of Polymeric

30 Crystallinity. Cryst Growth Des 16:3345-3352 doi:10.1021/acs.cgd.6b00315

31 Song G, Yu J, Ding M, Zhang J (2018) A Novel Cellulose/Ionic Liquid Complex Crystal. Cryst

32 Growth Des 18:4260-4264 doi:10.1021/acs.cgd.8b00754

33 Wada M, Kwon GJ, Nishiyama Y (2008) Structure and Thermal Behavior of a Cellulose

34 I-Ethylenediamine Complex. Biomacromolecules 9:2898-2904 doi:10.1021/bm8006709

35 Wada M, Nishiyama Y, Langan P (2006) X-ray Structure of Ammonia-Cellulose I: New Insights

36 into the Conversion of Cellulose I to Cellulose IIII. Macromolecules 39:2947-2952

37 doi: $10.1021 / \mathrm{ma} 060228 \mathrm{~s}$

38 Wertheim GK, Butler MA, West KW, Buchanan DNE (1974) Determination of the Gaussian and

39 Lorentzian content of experimental line shapes. Rev Sci Instrum 45:1369-1371

40 doi:10.1063/1.1686503 
1 Yamane C, Miyamoto H, Hayakawa D, Ueda K (2013) Folded-chain structure of cellulose II

2 suggested by molecular dynamics simulation. Carbohydr Res 379:30-37

3 doi:10.1016/j.carres.2013.06.012

4 Zhang H, Wu J, Zhang J, He JS (2005) 1-Allyl-3-methylimidazolium chloride room temperature

5 ionic liquid: A new and powerful nonderivatizing solvent for cellulose. Macromolecules 38:8272-

68277 doi:10.1021/ma0505676 